\title{
The anaesthetic management of a patient with Emery-Dreifuss muscular dystrophy
}

Valerie Jensen MD FRCP

Purpose: This case report presents a patient with EmeryDreifuss Muscular Dystrophy and describes the anaesthetic considerations.

Clinical features: The features of Emery-Dreifuss Muscular Dystrophy are contractures, humeroperoneal muscle weakness and cardiomyopathy. The anaesthetic considerations for this syndrome are difficult tracheal intubation, difficult spinal anaesthetic, heart block, gastric reflux, rhabdomyolysis, and unproved malignant hyperthermia susceptibility.

Conclusion: The major anaesthetic problem for the patient with Emery-Dreifuss Muscular Dystrophy could be a lifethreatening cardiomyopathy.

Objectif: Rapporter un cas de dystrophie musculaire d'EmeryDreyfuss et en décrire les conséquences pertinentes pour l'anesthésie.

Caractéristiques cliniques: La dystrophie musculaire d'EmeryDreyfuss est caractérisée par des contractures, une faiblesse des muscles huméraux et péronés et une cardiopathie. En face de ce syndrome, on doit s'attendre à une intubation trachéale difficile, une rachianesthésie laborieuse, un bloc cardiaque, un reflux oesophagéen, une rhabdomyolyse et une susceptibilité à l'hypothermie maligne (non prouvée).

Conclusion: Une cardiomyopathie potentiellement fatale constitue le problème anesthésique majeur de la dystrophie musculaire d'Emery-Dreyfuss.

Emery-Dreifuss Muscular Dystrophy (EDMD) is the third most common of the $\mathrm{X}$-linked recessive muscular dystrophies. It is characterized by early contractures, muscle weakness in a humeroperoneal distribution, and

\section{Key words}

COMPLICATIONS: muscular dystrophy, Emery-Dreifuss; ANAESTHETIC TECHNIQUES: spinal.

From the Department of Anaesthesia, 3B2-32 Walter Mackenzie Centre, University of Alberta Hospitals, 8440-112 St.,

Edmonton, Alberta, T6G 2B7.

Address correspondence to: Dr. V. Jensen.

Accepted for publication 13th May, 1996. cardiomyopathy. ${ }^{1}$ These features tend to develop independently of each other. ${ }^{2}$ Only one other case report of a patient with this disease has been reported in the English anaesthetic literature. ${ }^{3}$ The true frequency of EDMD is unknown ${ }^{4}$; it is less common than Becker Muscular Dystrophy (BMD), which has an incidence of 1 in 33,000 male births. ${ }^{5}$ Emery-Dreifuss Muscular Dystrophy was once considered to be a benign muscular dystrophy because the muscular problems are not lifethreatening, however it has been recently recognized that the cardiac problems present a risk to the patient's life. ${ }^{1-3,6}$

\section{Case report}

A 26-yr-old white male science graduate presented for a testicular biopsy as an outpatient. He had recently been diagnosed with EDMD. In his past history, he had started to walk at the usual age. He always had an awkward gait and an inability to straighten his elbows. He was unable to participate in sports that required running but he was proficient at swimming. His swimming coaches informed him that his degree of elbow flexion was abnormal. He received an uneventful general anaesthetic in South Africa at the age of $6 \mathrm{yr}$ for knee surgery. At the age of $15 \mathrm{yr}$ a muscle biopsy and elevated serum enzymes confirmed the diagnosis of muscular dystrophy.

His second general anesthetic was in Canada at the age of $18 \mathrm{yr}$ for an appendectomy. For this anaesthetic, the induction was accomplished with $0.1 \mathrm{mg}$ fentanyl, 3 mg d-tubocurarine, $150 \mathrm{mg}$ thiopentone, and $150 \mathrm{mg}$ succinylcholine. The details of the tracheal intubation were not recorded. The anaesthetic was maintained with a succinylcholine infusion, nitrous oxide, oxygen, and enflurane. The patient's blood pressure was $110 \mathrm{mmHg}$ systolic and his heart rate was $90 \mathrm{bpm}$ during the procedure. His temperature was $38.6^{\circ} \mathrm{C}$ in the recovery room but this was thought to be due to acute appendicitis, and the recovery period was uneventful.

When the patient was in his early twenties, an ECG showed a dysrhythmia termed atrial flutter, which had a regular rapid atrial tachycardia, an atrioventricular 
block, and a slower ventricular rate. Genetic testing became available and confirmed the diagnosis of EDMD.

On direct questioning, he denied any cramps, stiffness, or weakness of his muscles. He denied neck stiffness, gastric reflux, or a history of allergies. He admitted to smoking but he denied any respiratory symptoms. He denied any chest pain, dyspnea, orthopnea, or syncope. There was no family history of muscular or anaesthetric problems. His father and mother and two sisters were all well.

On physical examination he was of average height and weight, at $73 \mathrm{~kg}$ and $170 \mathrm{~cm}$. His airway was assessed to be Mallampati Class II, and there was no restriction of his neck movement. His blood pressure was $150 / 70 \mathrm{mmHg}$ and his heart rate was $50 \mathrm{bpm}$ and irregular. Auscultation of his heart revealed that the $S_{1}$ was accentuated, the $S_{2}$ was normal, and there was a grade $4 / 6$ systolic murmur at the apex. The jugular venous pulse was not elevated and flutter waves were observed at his neck. His respiratory system was normal. On examination of his neuromuscular system, the sensory examination was normal and his deep tendon reflexes were decreased. The biceps, triceps, calf, and quadriceps muscles were mildly atrophic. His muscular strength was normal and there was no muscular pseudohypertrophy. His lumbar spine displayed normal mobility. A preoperative ECG showed atrial flutter with a rate of $54 \mathrm{bpm}$.

The patient agreed to accept a spinal anaesthetic for the testicular biopsy. The monitors included an automated blood pressure cuff,. an .ECG monitor, and an oxygen saturation monitor. A 25 gauge Whitacre spinal needle was inserted at $\mathrm{L}_{4-5}$ while the patient was in the sitting position and $60 \mathrm{mg}$ hyperbaric lidocaine was administered. The blood pressure remained at $150 / 80 \mathrm{mmHg}$ and the heart rate at $52 \mathrm{bpm}$ during the $20 \mathrm{~min}$ procedure. The recovery period was uneventful and the patient was discharged several hours after the anaesthetic.

The cardiology service then investigated the cardiac status of the patient. An echocardiogram revealed normal left and right ventricular function. There was no evidence of thrombosis in the atria. There was mild mitral valve thickening with mitral and tricuspid regurgitation. A $24 \mathrm{hr}$ Holter monitor showed a variation in heart rate from 30 to $126 \mathrm{bpm}$. The decision was made to convert the heart rhythm from atrial flutter to sinus rhythm. Overdrive pacing with a temporary pacemaker was unsuccessful; therefore cardioversion was planned. Propofol $140 \mathrm{mg}$ was administered by an anaesthetist and a $200 \mathrm{~J}$ shock was given, which converted the atrial flutter to asystole. Temporary pacing for five minutes was required before junctional thythm returned with atrial standstill. After $20 \mathrm{~min}$, sinus rhythm returned. Because of the period of asystole and because of the natural history of the disease, the decision was made to insert a permanent VVI pacemaker set at a rate of 40 bpm. The patient has been asymptomatic for the following year.

\section{Discussion}

Emery-Dreifuss Muscular Dystrophy is a rare type of muscular dystrophy with, typically, an X-linked inheritance, although this case was sporadic. Duchenne Muscular Dystrophy (DMD) is the most common type of X-linked dystrophy, and BMD is the second most common. We will discuss the clinical and diagnostic features of EDMD and summarize the anaesthetic considerations.

The early appearance of contractures before the development of any muscle weakness is unique to EDMD. The contractures typically occur at the elbows, Achilles tendons, and postcervical muscles.' Some patients also develop contractures of the muscles of the lower back, making forward flexion of the spinal column almost impossible. ${ }^{2,7}$

The muscle wasting and weakness initially occur in a humeroperoneal distribution. Later weakness of the hip and knee extensors and the proximal upper limb musculature also develops. ${ }^{1,8}$ The patient usually remains ambulatory.

The severity of heart disease does not correlate with the degree of skeletal muscle involvement. Atrial conduction defects with a bradycardia are considered to be the hallmark of EDMD cardiomyopathy. ${ }^{2}$ In asymptomatic patients, an ECG may show low amplitude $P$ waves and a first degree heart block. These changes may progress to atrial fibrillation or flutter and then to second or third degree heart block with a slow junctional rhythm. Atrial standstill is a pathognomonic finding. ${ }^{7}$ A ventricular cardiomyopathy may develop later in the course of the disease. The rate of progression and severity of the cardiac disease is extremely variable. Death may be sudden or caused by congestive heart failure or complications of thromboemboli from the heart. ${ }^{7}$

In contrast to other dystrophies, female carriers of EDMD may harbour a considerable cardiac risk with advancing age even without manifesting muscular weakness or contractures. 6,7

The diagnosis of EDMD is confirmed by laboratory investigations including muscle biopsy and histology, electromyography, serum enzymes, and genetic testing. All of the muscular dystrophies share a common histology of affected muscle that is characterized by (to vary- 
ing degrees) variation in fibre size, fibre necrosis and phagocytosis, and ultimately replacement by fat and connective tissue.'

Elecromyographic examination of the affected muscle reveals typical myopathic features of low-amplitude and short duration potentials. ${ }^{4}$ Elevated serum creatine phosphokinase (CK) enzymes occur in the serum of males affected with EDMD, as evidence of chronic rhabdomyolysis. ${ }^{9}$ The CK peaks at approximately five times normal in childhood or adolescence and then declines slowly throughout adult life. ${ }^{10}$ The aldolase level may be normal in EDMD even though the CK levels are elevated. Such a disparity between the two muscle enzyme values is not typical of neuromuscular disease even though this occurs in EDMD ${ }^{4,10}$ Genetic testing confirms the diagnosis of EDMD. The disease gene of EDMD has been mapped to distal Xq28. The EDMD gene encodes a novel serine-rich protein termed emerin, which has features indicating that it may be a membrane spanning protein. ${ }^{11}$ Genetic mapping has localized the gene for both DMD and BMD to Xp21. ${ }^{12}$ The protein named dystrophin, which is reduced or absent in the Becker and Duchenne forms, has been found to be normal in EDMD.'

The following discussion will summarize some of the differences as well as the similarities in management between EDMD and the other two types of X-linked recessive muscular dystrophy. The general principles of anaesthetic management of the patient with DMD are elucidated in a review article. ${ }^{13}$ Respiratory function is usually maintained in the patient with EDMD, because any scoliosis usually stabilizes spontaneously. ${ }^{8}$ Conversely, the patient with DMD has increasing respiratory insufficiency with advancing age, as the truncal muscles become weaker. ${ }^{12,13}$ Scoliosis may become severe in the patient with DMD as the patient becomes wheelchair bound..$^{12}$ Deterioration in respiratory function after anaesthesia has been reported in the patient with $\mathrm{DMD}^{13}$ but this complication is not as likely to occur in the patient with EDMD. Flexion contractures of the neck and, less commonly, the lumbar paravertebral muscles in the patient with EDMD may pose anatomical difficulties for endotracheal intubation ${ }^{3}$ and for administration of a spinal anaesthetic respectively. With DMD the skeletal muscle weakness predominates and muscle contractures are secondary to the muscle weakness. Usually, there is no anticipated difficulty with tracheal intubation in the patient with DMD. However, a possible scoliosis would increase the difficulty of locating the intrathecal space.

There is no severe intellectual deficit associated with EDMD. ${ }^{3}$ Mental impairment may be present in up to $30 \%$ of DMD patients. ${ }^{12}$ The presence of mental impair- ment makes it more difficult for the patient to comply with acceptance of the anaesthetic.

The cardiac status of a patient with EDMD should be evaluated to determine the necessity for preoperative pacemaker insertion. ${ }^{3}$ In Smith's review, there was no mention that a pacemaker may be useful for the management of the patient with DMD. ${ }^{13}$ In contrast to the bradycardia and prolonged P-R interval in the ECG of the patient with EDMD, ${ }^{4,13}$ tachycardia and a shortened P-R interval are manifested with DMD ${ }^{13}$ A cardiomyopathy localized to the posterobasal segment of the left ventricle is usual in DMD. ${ }^{14}$ The patient with EDMD may have generalized cardiomyopathy, with the atria showing earlier and more severe involvement. ${ }^{14}$

Delayed gastric emptying has been reported in the patient with DMD. ${ }^{13,15}$ Deficient dystrophin may lead to visceral smooth muscle involvement of the gastrointestinal tract. ${ }^{15}$ It is not known whether deficient emerin in the patient with EDMD could also lead to the same problem. A six hour preoperative fast and treatment with a prokinetic agent reduce the risk of pulmonary aspiration in the patient with an X-linked muscular dystrophy.

Rhabdomyolysis after succinylcholine leading to cardiac arrest has been reported in patients who were later diagnosed to have BMD or DMD. ${ }^{5,16}$ The absence of an elevated end-tidal $\mathrm{CO}_{2}$ and the response to therapy that did not include dantrolene distinguish rhabdomyolysis from malignant hyperthermia. Some evidence exists that patients with muscle disorders with normal dystrophin do not get anaesthesia-induced rhabdomyolysis. ${ }^{16}$ The anaesthetist should be aware that acute rhabdomyolysis in the perioperative period can be induced by other factors besides succinylcholine, including general anaesthetics and electrolyte disorders. 9.17

Malignant hyperthermia possibly is associated with DMD and other myopathies; ${ }^{18}$ although genetic linkage between malignant hyperthermia and the X-linked dystrophies is unlikely. ${ }^{19}$ The suggestion has been made that triggering anaesthetics should be avoided in the patient with EDMD, ${ }^{3}$ but this is not based on substantial evidence.

\section{Conclusion}

Careful preoperative assessment of the patient with EDMD is essential, because the cardiac problems may be considerable even if the contractures and muscle weakness are minimal. A regional or local anaesthetic would reduce the incidence of the problems of drug interactions, rhabdomyolysis, and malignant hyperthermia. The type of anaesthetic should be adapted to the individual patient and to the proposed surgical procedure. 


\section{Acknowledgement}

The author wishes to thank Dr. Brendan Finucane for previewing the manuscript.

\section{References}

1 Emery $A E H$. Emery-Dreifuss muscular dystrophy and other related disorders. Br Med Bull 1989; 45: 772-87.

2 Voit T, Krogmann $O$, Lenard HG. Emery-Dreifuss muscular dystrophy: disease spectrum and differential diagnosis. Neuropediatrics 1988; 19: 62-71.

3 Morrison P, Jago RH. Emery-Dreifuss muscular dystrophy. Anaesthesia 1991; 46: 33-5.

4 Case records of the Massachusetts General Hospital. Weekly clinicopathological exercises. N Engl J Med 1992; 327: 548-57.

5 Sullivan $M$, Thompson $W K$, Hill GD. Succinylcholineinduced cardiac arrest in children with undiagnosed myopathy. Can J Anaesth 1994; 41: 497-501:

6 Dickey RP, Ziter FA, Smith RA. Emery-Dreifuss muscular dystrophy. J Pediatr 1984; 104: 555-9.

7 Fishbein MC, Siegel RJ, Thompson CE, Hopkins LC. Sudden death of a carrier of X-linked Emery-Dreifuss muscular dystrophy. Ann Intern Med 1993; 119: 900-5.

8 Shapiro F, Specht L. Orthopedic deformities in EmeryDreifuss muscular dystrophy. J Pediatr Orthop 1991; 11 : 336-40.

9 Brumback RA, Feeback DL, Leech RW. Rhabdomyolysis in childhood. A primer on normal muscle function and selected metabolic myopathies characterized by disordered energy production. Pediatr Clin North Am 1992; 39: 821-58.

10 Bialer MG, Bruns DE, Kelly TE. Muscle enzymes and isoenzymes in Emery-Dreifuss muscular dystrophy. Clin Chem 1990; 36: 427-30.

11 Bione S, Maestrini E, Rivella S, et al. Identification of a novel X-linked gene responsible for Emery-Dreifuss muscular dystrophy. Nature Genetics 1994; 8: 323-7.

12 Iannaccone ST. Current status of Duchenne muscular dystrophy. Pediatr Clin North Am 1992; 39: 879-94.

13 Smith CL, Bush GH. Anaesthesia and progressive muscular dystrophy. Br J Anaesth 1984: 57: 1113-8.

14 Bialer MG, McDaniel NL, Kelly TE. Progression of cardiac disease in Emery-Dreifuss muscular dystrophy. Clin Cardiol 1991; 14: 411-6.

15 Staiano A, Del Giudice E, Romano A, et al. Upper gastrointestinal tract motility in children with progressive muscular dystrophy. J Pediatr 1992; 121: 720-4.

16 Farrell PT. Anaesthesia-induced rhabdomyolysis causing cardiac arrest: case report and review of anaesthesia and the dystrophinopathies. Anaesth Intensive Care 1994; 22 : 597-601.

17 Curry SC, Chang D, Connor D. Drug- and toxin -induced rhabdomyolysis. Ann Emerg Med 1989; 18: 1068-84.
18 Brownell $A K W$. Malignant hyperthermia: relationship to other diseases. Br J Anaesth 1988; 60: 303-8.

19 Iaizzo PA, Palahniuk RJ. Malignant hyperthermia. Diagnosis, treatment, genetics, and pathophysiology. Invest Radiol 1991; 26: 1013-8. 